\title{
New restrictions on celecoxib (Celebrex) use and the withdrawal of valdecoxib (Bextra)
}

Published at www.cmaj.ca on Apr. 15, 2005. Revised on Apr. 19, 2005.

Reason for posting: Coxibs, the class of NSAIDs that selectively inhibit cyclooxygenase 2 (COX-2), were designed to reduce joint pain and inflammation without causing the gastric epithelial adverse effects typical of nonselective NSAIDs. Rofecoxib (Vioxx) was withdrawn from the market in September 2004 over concerns about cardiovascular adverse effects, and key safety trials involving celecoxib (Celebrex) ${ }^{1}$ and valdecoxib $(\text { Bextra })^{2}$ have recently been published. Health Canada now recommends new restrictions on celecoxib use, and valdecoxib has been taken off the market. The US Food and Drug Administration has gone further, directing that all prescription and over-the-counter NSAIDs include specific information regarding potential cardiovascular, gastrointestinal and other risks.

The drugs: The results of recent trials have raised concerns that coxib use increases the risk of cardiovascular adverse events. In the APC study, a trial on colorectal adenoma prevention, 2035 patients were randomly assigned to celecoxib $200 \mathrm{mg}$ twice daily or $400 \mathrm{mg}$ twice daily or placebo. After about 3 years, cardiovascular events (death from cardiovascular causes, myocardial infarction, stroke, heart failure) had occurred in $1 \%$ of patients receiving placebo, $2.3 \%$ of the group taking $200 \mathrm{mg}$ celecoxib (hazard ratio [HR] 2.3, 95\% confidence interval [CI] 0.9-5.5), and 3.4\% (HR 3.4, 95\% CI 1.4-7.8) of the group taking $400 \mathrm{mg} .{ }^{1}$

In a trial involving $1671 \mathrm{pa}-$ tients who underwent coronary artery bypass grafting, cardiovascular events were more frequent among those taking valdecoxib and parecoxib for pain than among those taking placebo $(2 \%$ v. $0.5 \%$; risk ratio $3.7,95 \% \mathrm{CI}$ 1.0-13.5). ${ }^{2}$ Amid concerns about reports of severe cutaneous reactions (Stevens-Johnson syndrome, erythema multiforme, toxic epidermal necrolysis) among patients taking valdecoxib, ${ }^{3}$ the drug was removed from the market.

What to do: COX-2 inhibitors appear to increase the risk of cardiovascular adverse events in a dose-related fashion, and all patients should be informed of this. Calculating the patient's baseline risk of cardiovascular disease (e.g., with Framingham risk calculators) may be wise, and celecoxib should not be prescribed to patients with cardiovascular disease or diabetes or those at increased risk of cardiovascular events. Celecoxib should be used in the lowest effective doses for short periods (weeks) only. A risk-benefit discussion is necessary for those requiring the drug for a longer period. Of note, patients in the APC study ${ }^{1}$ who used celecoxib for 3 years at a dose double that recommended for osteoarthritis had an increase in absolute risk of cardiovascular events of about $1.3 \%$ (number needed to harm of 77).

The only NSAID known to reduce primary and secondary cardiovascular events is ASA. The absolute risk of other NSAIDs is unclear. Appreciating the relative degree of COX-2 selectivity of some commonly used NSAIDs
Table 1: The degree of inhibition of COX-2 relative to COX-1 for various NSAIDs

\begin{tabular}{lc}
\hline NSAID type & COX-2 selectivity* \\
\hline COX-2 selective inhibitors & \\
Rofecoxib & 80 \\
Etodolac & 23 \\
Meloxicam & 11 \\
Celecoxib & 9 \\
Nonselective NSAIDs & \\
Diclofenac & 4 \\
Sulindac & 3 \\
Piroxicam & 2 \\
Ibuprofen & 0.4 \\
Naproxen & 0.3 \\
Indomethacin & 0.2 \\
Ketorolac & 0.003 \\
\hline
\end{tabular}

Note: $\mathrm{COX}=$ cyclooxygenase.

*The $80 \%$ inhibitory concentration ratios of COX-2 relative to COX-1 in human whole blood assays. ${ }^{6}$

(Table 1) may be useful. Alternative pharmacologic and nonpharmacologic approaches to pain management should be reviewed and strongly considered.

\section{Jill Cotter}

Resident

Department of Family Medicine

University of Ottawa

Eric Wooltorton

CMAF

\section{References}

1. Solomon SD, McMurray JJ, Pfeffer MA, Wittes J, Fowler R, Finn P, et al. Cardiovascular risk associated with celecoxib in a clinical trial for colorectal adenoma prevention. $N$ Engl 7 Med 2005;352:1071-80.

2. Nussmeier NA, Whelton AA, Brown MT, Langford RM, Hoeft A, Parlow $\mathrm{JL}$, et al. Complications of the Cox-2 inhibitors parecoxib and valdecoxib after cardiac surgery. $N$ Engl $7 \mathrm{Med}$ 2005;352:1081-91.

3. Health Canada. Health Canada endorsed important safety information on BEXTRA (valdecoxib) tablets [letter]. Dec. 10, 2004. Available at: www.hc-sc .gc.ca/hpfb-dgpsa/tpd-dpt/bextra2_hpc _e.html (accessed 2005 Apr 14). 